\title{
Über die Fettsäuren der Cholesterinester aus menschlichen Nebennieren
}

\author{
Von \\ D. Eberhagen und N. ZöLLNER \\ Aus der Mediziniscken Poliklinik der Universisät München (Direktor: Prof. Dr. med. W. Seitz.)
}

(Der Schriftleitung zugegangen am 30. Mai 1963)

\begin{abstract}
Es wurde die Fettsäurenzusammensetzung der Cholesterinester von 8 menschlichen Nebennieren gaschromatographisch untersucht. Vorherrschende Fettsäure ist die Olsäure. Bemerkenswert ist der große Anteil der Polyenfettsäuren. Bei den als Normalorgane angesehenen Nebennieren ergab sich ein auffallend hoher Gehalt an Dokosatetraensäure und an Eikosatriensäure. Die Arachidonsäurewerte betrugen nur etwa die Hälfte bis Zweidrittel der der Eikosatriensäure. Die Konzentration der Linolsäure lag in der Regel zwischen der der Arachidonsäure und der der Eikosatriensäure. Histologisch entspeicherte Nebennieren wiesen eine andere Fettsäurezusammensetzung mit höherem Arachidonsäure- und erniedrigtem Eikosatriensäuregehalt auf.
\end{abstract}

The fatty acid composition of the cholesterol esters of 8 human adrenals was studied with gas chromatography. The predominant fatty acid was oleic acid. There was a remarkably high proportion of polyenoic acids. In adrenals, which were considered normal, there was a markedly high content of docosatetracnoic acid and eicosatrienoic acid. The level of arachidonic acid was only about one half to two thirds that of the eicosatrienoic acid. As a rule, the concentration of linoleic acid was between those of arachidonic and eicosatrienoic acid. Histologically defatted adrenals had a different fatty acid composition, with higher arachidonic and decreased eicosatrienoic acid contents.

Die Fettsäuren der Nebennierenlipoide zeigen in ihrer Zusammensetzung gegenüber denen in anderen Organen gewisse Besonderheiten. So war früher die Nebenniere wegen ihres relativ hohen Arachidonsäuregehaltes eine bevorzugte Quelle zur Gewinnung dieser Verbindung (1). Andererseits konnte bisher weder in tierischen (2) noch in menschlichen (3) Nebennieren ein Anhalt für das Vorhandensein einer $\mathrm{C}_{22}$-Hexaensäure erbracht werden, die sich sonst in zwar kleinen, aber immer einwandfrei erfaßbaren Mengen in fast allen Organen und auch im Blutserum nachweisen läßt. Bei der vorliegenden Untersuchung der Cholesterinester, deren Gehalt in den Nebennieren in Abhängigkeit vom Funktionszustand der Organe großen Schwankungen unterliegt (4), ergaben sich in der Fettsäurezusammensetzung ebenfalls einige auffällige Abweichungen von den sonst üblichen Verhältnissen.

Zur Aufarbeitung gelangten die nach FolCH und Mitarbeitern (5) extrahierten Gesamtlipoide von 8 Nebennieren. Die Nebennieren waren bei der Sektion Leichen beiderlei Geschlechts und verschiedenen Alters entnommen worden $\left.{ }^{1}\right)$. Durch Abtrennung der polaren Lipoidanteile mit Hilfe der Gummimembrandialyse (6) und der Glyceride sowie des freien Cholesterins auf chromatographischem Wege ließen sich die Cholesterin= ester darstellen. Die gaschromatographisch ermittelte Zusammensetzung ihrer durch Umestẹrung gewonnenen Fettsäuren in Prozenten der Gesamtfettsäuren gibt die Tabelle 1 wieder.

1) Die Präparation und histologische Untersuchung der Organe wurde von Herrn Dr. F. Lindicar, Pathologisches Institut der Freien Universität Berlin vorgenommen, dem wir herzlich danken möchten.
Am bemerkenswertesten erscheint der hohe Eikosatriensäuregehalt, wie er sich sonst vorzugsweise in den Lipoidextrakten von Tieren findet, die mit einer polyensäurefreien Diät aufgezogen sind (7). Ob auch hier als Hauptvertreter der $C_{20}$-Triensäure das $\triangle^{5,8,11}$-Isomere vorliegt, das sich in vielen Fällen als der überwiegende Bestandteil dieses Isomerengemisches erwiesen hat, kann nicht entschieden werden. Auffallend sind auch die von zwei Ausnahmen abgesehen - niedrigen Eikosatetraensäure- (Arachidonsäure-) und Oktadekadiensäure(Linolsäure-)anteile, die unter den $\mathrm{C}_{20}$-Triensäurewerten liegen. Bei den Nebennieren 7 und 8, die einen höheren Arachidonsäuregehält besitzen, handelt es sich histologisch um entspeicherte Organe. Bezieht man die Prozentzahlen auf die Gesamtlipoidwerte, so ergeben sich sowohl für die $\mathrm{C}_{20^{-}}$wie auch für die $\mathrm{C}_{22}$-Tetraensäuren relativ konstante Werte für alle untersuchten Organe. Dafür findet sich jetzt bei den gesättigten und einfach ungesättigten Fettsäuren eine außerordentliche Variabilität der Prozentzahlen. Unerwartet hohe Anteile sind auch für die Dokosatetraensäure nachgewiesen. Diese Ergebnisse stimmen in mancher Hinsicht nicht mit den entsprechenden Befunden von DAILEY und Mitarbeitern (8) überein, die vor allem nicht über einen derart hohen $\mathrm{C}_{20}$-Triensäure- und $\mathrm{C}_{22}$-Tetraensäuregehalt berichteten.

Wie weit die in Abhängigkeit vom histologischen Bild stehende, besondere Fettsäurenzusammensetzung der Cholesterinester mit der Organfunktion zusammenhängt, müssen weitere Untetsuchungen ergeben. Verschiedene japanische Autoren (9) haben kürzlich über Versuche berichtet, in denen sie dic Fettsäurenzusammensetzung der Nebennierenlipoide diätetisch und hormonal stark verändern konnten. 
Tab. 1. Zusammensetzung der Cholesterinestcrfettsäuren von menschlichen Nebennieren in Prozent der Gesamtfettsäuren

\begin{tabular}{|c|c|c|c|c|c|c|c|c|c|}
\hline \multirow{2}{*}{$\begin{array}{l}\text { Zehl der } \\
\text { C-Atome }\end{array}$} & \multirow{2}{*}{$\begin{array}{c}\text { Zahl d. } \\
\text { Doppel- } \\
\text { bindungen }\end{array}$} & \multicolumn{8}{|c|}{ Nebenniere") } \\
\hline & & 1 & 2 & 3 & 4 & 5 & 6 & 7 & 8 \\
\hline$\leqq 10$ & 0 & + & 1,5 & 0,5 & & & & 0,5 & + \\
\hline 12 & 0 & 0,5 & + & & + & 0,5 & + & + & 0,5 \\
\hline 14 & 0 & 3,0 & 2,5 & 4,0 & 3,0 & 4,5 & 2,5 & 2,5 & 1,5 \\
\hline 15 & 0 & 0,5 & 0,5 & 0,5 & 0,5 & 0,5 & 0,5 & 0,5 & 0,5 \\
\hline 16 & 0 & 2,5 & 8,0 & 7,5 & 2,5 & 10,0 & 9,0 & 5,5 & 6,0 \\
\hline 16 & 1 & 3,5 & 4,5 & 4,0 & 6,0 & 6,0 & 5,0 & 4,5 & 3.5 \\
\hline 17 & 0 & 0,5 & 0,5 & 0,5 & 0,5 & 0,5 & 0,5 & 1,5 & 1,0 \\
\hline 18 & 0 & 5,0 & 2,5 & 1,5 & 2,5 & 3,0 & 2,0 & 1,0 & 3,0 \\
\hline 18 & 1 & 41,5 & 40,0 & 38,0 & 32,0 & 38,0 & 39,0 & 31,0 & 25,5 \\
\hline 18 & 2 & 6,0 & 6,5 & 4,0 & 4,0 & 5,0 & 3,5 & 4,5 & 4,5 \\
\hline 19 & o & 0,5 & & 0.5 & 0,5 & 0,5 & + & 0,5 & 0,5 \\
\hline 20 & 0 & & + & + & + & 1,0 & 1,0 & 0,5 & 0,5 \\
\hline 20 & 1 & 3,0 & 3,5 & 4,0 & 3,5 & 6,5 & 3,0 & 3,0 & 3,5 \\
\hline 20 & 2 & 1,5 & 2,0 & 3.0 & 3,0 & 2,5 & 1,5 & 2,0 & 1,5 \\
\hline 20 & 3 & 5,5 & 7,5 & 6,0 & 5,0 & 4,5 & 6,0 & 6,5 & 3,5 \\
\hline 20 & 4 & 4,0 & 4,5 & 3,0 & 2,5 & 2,0 & 4,5 & 6,0 & 9,0 \\
\hline 20 & 5 & 1,0 & + & & 1,0 & $?$ & + & + & + \\
\hline 22 & 0 & & & & + & + & & 0,5 & 0,5 \\
\hline 22 & 1 & & 1,5 & 2,5 & 4,0 & 1,5 & 2,0 & 2,0 & 2,5 \\
\hline 22 & 2 & 2,0 & 1,0 & 1,5 & 1,0 & + & 0,5 & 1,5 & 1,5 \\
\hline 22 & 3 & 1,5 & 1,0 & 1,5 & 2,5 & 0,5 & 2.5 & 2,0 & 3,5 \\
\hline 22 & 4 & 1,0 & 7,5 & 9,0 & 11,5 & 7,5 & 9.5 & 11,5 & 17,5 \\
\hline 22 & 5 & 0,5 & 1,5 & 2,5 & 1,0 & 1,5 & 1,5 & 4,5 & 6,0 \\
\hline 24 & $?$ & + & 1,5 & 2,5 & 1,5 & 1,0 & 2,5 & 2,0 & + \\
\hline
\end{tabular}

-) Die Nebennieren 1-5 waren histologisch im wesentlichen normal, 6-8 in zunehmendem Maße entspeichert.

\section{Versuche}

Die Nebennieren wurden unmittelbar nach der Entnahme vom anhaftenden Fettgewebe frei präpariert, zerkleinert und nach Folch (5) mit Chloroform-Methanol extrahiert. Die eingedampften Lipoidextrakte wurden ebenso wie die bei $110^{\circ}$ bis zur Gewichtskonstanz getrockneten Geweberückstände gewogen. Die Entfernung der Phosphatide und ähnlich polarer Bestandteile aus dem Lipoidauszug geschah durch Dialyse der Neutralfette und des Cholesterins in petrolätherischer Lösung durch eine dünne Gummimembran hindurch (6b). Zur Gewinnung der Cholesterinester wurde das Dialysat auf ein mit $20 \mathrm{~g}$ Florisil (Fluka) gefülltes Chromatographierohr $(25 \times 1,6 \mathrm{~cm})$ gegeben und die Fettstoffe mit $300 \mathrm{~m} l$ Petroläther (Vorlauf), $600 \mathrm{ml} 3 \%$ Äther in Petroläther (Cholesterinester) und schließlich mit $400 \mathrm{ml} \mathrm{10 \%} \mathrm{Methanol} \mathrm{ent-}$ haltendem Äther (Glyceride, Cholesterin) eluiert. Die Tabelle 2 gibt die erhaltenen Ausbeuten wieder. $\mathrm{Da}$ die Spaltung der Cholesterinester mit methanolischer $\mathrm{NaOH}$ oder $\mathrm{HCl}$ selbst nach langen Reaktionszeiten und erhöhten Ionenkonzentrationen in folge der schlechten Löslichkeit der Ester nur zu ungenügenden Ausbeuten führt (10), löste man sie zuerst in Methylacetat $(20 \mathrm{~m} /$
Tab. 2. Gesamtlipoid- und Cholesterinester-Ausbeuten aus Nebennieren (Werte in g)

\begin{tabular}{|c|c|c|c|c|c|c|c|c|}
\hline & \multicolumn{8}{|c|}{ Nebenniere } \\
\hline & 1 & 2 & 3 & 4 & 5 & 0 & 7 & 8 \\
\hline Organfrischgewicht & 4,11 & 8,86 & 7,26 & 4,76 & 3,11 & 6,76 & & 4,40 \\
\hline Trockensubatanx & 1,146 & 2,733 & 1,528 & 1,087 & 0,699 & 1,368 & 0,990 & 0,775 \\
\hline Gesamtlipoide & 0.595 & 1,705 & 0,674 & 0,494 & 0,258 & 0,417 & 0,395 & 0,210 \\
\hline Cholesterinesser & 0,369 & 0,833 & 0,285 & 0,200 & 0,110 & 0,075 & 0,088 & 0,029 \\
\hline $\begin{array}{l}\text { Cholesterinester in } \\
\% \text { der Gesamelipoide }\end{array}$ & 62,0 & 48,9 & 42,3 & 40,4 & 42,6 & 18,0 & 22,3 & 13,8 \\
\hline
\end{tabular}

Methylacetat pro $50 \mathrm{mg}$ Substanz) und setzte dann eine wasserfreie 5proz. methanolische Salzsäure $(40 \mathrm{~m} l$ pro $20 \mathrm{ml}$ Methylacetat) hinzu. Dabei kam es gewöhnlich zur Trübung der Lösung, die aber beim Erhitzen sofort wieder verschwand. Die Lösungen wurden jeweils etwa $90 \mathrm{Min}$. bei $70^{\circ}$ unter Rückfluß gekocht und nach dem Erkalten die Fettstoffe mit Petroläther in üblicher Weise ausgezogen, nachdem pro $60 \mathrm{~m} l$ Lösung $20 \mathrm{~m} l$ Wasser zugegeben waren. Es ließen sich dünnschichtchromatographisch (11) in keinem Fall Cholesterinester mehr nachweisen. Über eine zweite Florisil-Säule wurden mit der oben angegebenen Lösungsmittelfolge die Fettsäuremethylester gewonnen. - Die gaschromatographische Untersuchung der Fettsäuremethylester und die Berechnung der Zusammensetzung geschah in der gleichen Weise wie bereits früher beschrieben (10). Außerdem wurden die UVSpektren der verschiedenen nach HoLMaN und BuRR (12) alkaliisomerisierten Proben aufgenommen. Die Abbildung 1 gibt ein typisches Beispiel für die dabei ermittelten Kurvenverläufe wieder.

Frl. G. v. HELDEN danken wir für ihre verständnisvolle Mitarbeit.

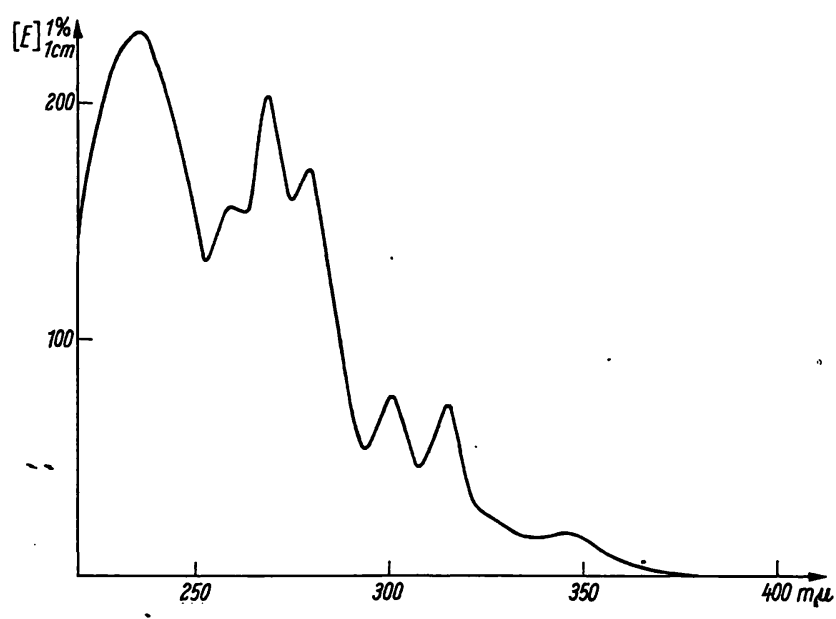

\section{Literatur}

1. Ault, W. C. und J. B. Brown, J. biol. Chemistry 107, 607 und 615 (1934); WhITE, M. F. und J. B. Brown, J. Amer. chem. Soc. 70, 4269 (1948). - 2. Klenk, E. und D. Eberhagen, HoppeSeyler's Z. physiol. Chem. 322, 258 (1960); Chang, T.-C. L. und C. C. SweELEy, J. Lipid Res. 3, 170 (1962). - 3. Unveröffentlichte Befunde. - 4. Deuel, jr., H. J., The Lipids, Bd. II, S. $765 \mathrm{ff}$., Interscience Publishers, Inc., New York-London (1955). - 5. Folch, J., M. Lees und G. H. S. StanLex, J. biol. Chemistry 226, 497 (1957). - 6a. van BeERs, G. J., H. DE IONGH und J. BOLDINGH in H. M. SinclaIr, Essential Fatty Acids, S. 43, Butterworth Scientific Publ., London (1958). - 6b. EBERHAGEN, D. und H.
Betzing, J. Lipid Res. 3, 382 (1962). - 7. Literatur s. KLENK, E. und K. Oetre, Hoppe-Seyler's Z. physiol. Chem. 318, 86 (1960). 8. Dailey, R. E., L. Swell, H. Field jr. und C. R. Treadwell, Proc. Soc. exp. Biol. Med. 105, 4 (1960). - 9. Nagase, M., Arch. jap. Chir. 29, 67 (1960); TAmakr, M., Arch. jap. Chir. 30, 611 (1961); Nakashio, S., Arch. jap. Chi1. 31, 48 (1962); Kumano, M. Arch. jap. Chir. 31, 115 (1962). - 10. Eberhagen, D., HoppeSeyler's Z. physiol. Chem. 333, (1963), (im Druck.) - 11. ZöLLNER, N. und G. Wolfram, Klin. Wschr. 40, 1101 (1962). - 12. HolmaN, R. T. und G. O. BurR, Arch. Biochem. Biophysićs 19, 474 (1948).

Prof. Dr. med. Nepomuk Zöllner Medizinische Poliklinik der Universität München 8 München 15, Pettenkoferstr. 8a 